\title{
Upregulation of the checkpoint protein CHFR is associated with tumor suppression in pancreatic cancers
}

\author{
DI ZHANG ${ }^{1}$, XIAO-LAN XU ${ }^{2}$, FEI LI ${ }^{1}$, HAI-CHEN SUN ${ }^{1}$, YE-QING CUI ${ }^{1}$, SHUANG LIU ${ }^{1}$ and PING-YONG XU ${ }^{2}$ \\ ${ }^{1}$ Department of General Surgery, Xuanwu Hospital, Capital Medical University, Beijing 100053; ${ }^{2}$ National Key Laboratory of \\ Biomacromolecules, Institute of Biophysics, Chinese Academy of Sciences, Beijing 100101, P.R. China
}

Received March 28, 2017; Accepted September 27, 2017

DOI: $10.3892 /$ ol.2017.7239

\begin{abstract}
The checkpoint with forkhead-associated (FHA) domain and RING-finger (CHFR) protein was identified as a cell cycle checkpoint protein and E3 ubiquitin ligase. In the present study, the potential functions of CHFR in pancreatic cancer were investigated. CHFR expression was measured in five pancreatic cancer cell lines by reverse transcriptionquantitative polymerase chain reaction and western blotting. Capan-1 cells stably expressing CHFR were established by lentiviral vector transfection. Cell proliferation was assessed using Cell Counting Kit-8, and cell migration/invasion assay was determined using Transwell assays. Cell cycle and apoptosis induced by gemcitabine or docetaxel were evaluated using flow cytometry. CHFR expression levels were also evaluated in pancreatic ductal adenocarcinoma (PDAC) tumor samples as well as adjacent non-tumor tissues by immunohistochemistry. The significance of CHFR expression was determined, with respect to clinicopathological features and overall survival. Overexpression of CHFR in Capan-1 cells led to a decreased proliferative rate and reduced cell migration and invasion abilities. Results also indicated an increase in G1 phase cells in Capan-1 cells overexpressing CHFR. Docetaxel-induced apoptosis was inhibited in Capan-1 cells with CHFR-overexpression. A reduction in CHFR expression was detected in $51.9 \%$ of patients with PDAC, which significantly correlated with later T-stage. The results show CHFR functions as a tumor suppressor in pancreatic cancer, suggests its potential role in controlling the cell cycle of pancreatic cancer cells; however, CHFR overexpression is not a favorable factor in apoptosis induced by docetaxel.
\end{abstract}

Correspondence to: Professor Fei Li, Department of General Surgery, Xuanwu Hospital, Capital Medical University, 45 Changchun Street, Beijing 100053, P.R. China

E-mail: feili36@aliyun.com

Key words: checkpoint with forkhead and ring-finger, pancreatic cancer, tumor suppressor, G1 arrest, drug resistance

\section{Introduction}

Pancreatic ductal adenocarcinoma (PDAC) is one of the most deadly types of cancer and the seventh leading cause of cancer death in both sexes. The overall five-year survival rate is about $6 \%$ (range from 2 to $9 \%$ ) (1). It is a highly malignant digestive system tumor with characteristic features of late discovery, early metastasis, rapid progress, and poor prognosis. Surgical resection is currently the only effective treatment, but only $20 \%$ of PDAC patients are eligible for operation when diagnosed (2). Earlier diagnosis and earlier treatment of pancreatic cancer have thus become particularly important. However, neither reliable screening tests for early diagnosis nor useful biomarkers to predict treatment efficacy is currently available $(3,4)$. Although research in this field has increased significantly over the past decade, no consistent conclusion has been reached. Therefore, it is necessary to find biomarkers that can help to diagnose pancreatic cancer at early stage, predict prognosis, or predict response to chemotherapeutic drugs.

Checkpoint with FHA domain and RING-finger (CHFR) was initially identified in a screen to find novel mitotic checkpoint proteins (5). The CHFR protein is a 664 amino acid protein with forkhead-associated (FHA) and RING-finger domains within its amino terminus and a cysteine-rich region within its carboxy terminus, which is very highly conserved between humans and mice (6-8). FHA domain is a phosphothreonine-binding domain, which was frequently found in DNA repair and checkpoint proteins $(6,9)$. Together previous studies indicate that, as a checkpoint protein, CHFR might play different roles at different phases of cell cycle, although there has been no unified conclusion $(5,10,11)$. Yu et al (10) firstly indicated CHFR was a tumor suppressor by the creation of CHFR knockout mouse. The study demonstrated that CHFR was important for maintaining genomic stability. However, another study in colon cancers was not arriving at the same conclusion (12). Last but not least, among the studies focused on the relationship between CHFR expression and the sensitivity to chemotherapeutics, inconsistent conclusions can also be found in different kinds of cancers (13-15). Consequently, the role of CHFR in different cancers seem to be different. So far, there is no published investigation focused on the role of $\mathrm{CHFR}$ in pancreatic cancer.

In the present study, the expression of CHFR was examined in several pancreatic cancer cell lines. We explored the 
function of CHFR in pancreatic cancer cells in vitro by examining the effects of altered CHFR expression on the malignant biological behaviors and chemotherapy sensitivity. Meanwhile, in order to investigate the potential of targeting CHFR as an index to predict prognosis, we also examined the associations among CHFR expression level, clinicopathologic parameters, and survival rate in PDAC patients.

\section{Materials and methods}

Cell culture and transfection. The pancreatic cancer cell lines CFPAC-1, BxPC-3, Capan-1, PANC-1, and SW1990 were purchased from the Cell Bank of the Chinese Science Academy (Shanghai, China) and cultured in the appropriate media (CFPAC-1, BxPC-3 and Capan-1 cells in RPMI-1640, Gibco; Thermo Fisher Scientific, Inc., Waltham, MA, USA; PANC-1 cells in DMEM; Thermo Fisher Scientific, Inc.; and SW1990 cells in L-15; Thermo Fisher Scientific, Inc.) supplemented with $10 \%$ fetal bovine serum (FBS; Thermo Fisher Scientific, Inc.) and $1 \%$ penicillin/streptomycin (Thermo Fisher Scientific, Inc.). To generate a stable Capan-1 cell line with CHFR overexpression, the complete human cDNA sequence of CHFR (NM_001161344) was cloned into the GV358 vector backbone downstream from an Ubi promoter (Ubi-CHFR-MCS-3FLAG-EGFP-IRES-piromycin) to produce GV358-CHFR vector, which carries the enhanced green fluorescent protein (EGFP) receptor gene. GV358 empty vectors were used to generate control cells. The GV358-CHFR vector and control vector were designed and synthesized by Genechem Co., Ltd. (Shanghai, China). Capan-1 cells were transfected with the appropriate lentiviral vector at a multiplicity of infection $(\mathrm{MOI})=25$ in medium containing $5 \mu \mathrm{g} / \mathrm{ml}$ polybrene (Genechem Co., Ltd.) according to the manufacturer's protocol. After $72 \mathrm{~h}$ of transfection, the medium was replaced with $2 \mathrm{ml}$ complete culture medium. Cells were selected with $0.2 \mu \mathrm{g} / \mathrm{ml}$ puromycin (Genechem Co., Ltd.) for $48 \mathrm{~h}$ and maintained until experimental analyses. The stable cell lines were confirmed by reverse transcription-quantitative polymerase chain reaction (RT-qPCR) and western blot analysis.

$R T$-qPCR. Total RNA was extracted with RNAiso Plus (\#9108; Takara Bio, Inc., Kusatsu, Japan) according to the manufacturer's recommended protocol and then reverse transcribed to cDNA using the Prime Script ${ }^{\mathrm{TM}}$ RT reagent kit with gDNA Eraser (\#RR047; Takara Bio, Inc.). Real-time amplification was carried out with a LightCycler ${ }^{\circledR} 480$ system (Roche Molecular Diagnostics, Pleasanton, CA, USA) and the product was quantified using an intercalating dye $\left(\mathrm{SYBR}^{\circledR}\right.$ Premix Ex Taq ${ }^{\mathrm{TM}} \mathrm{II}$; \#RR820A; Takara Bio, Inc.) that exhibits an increased fluorescence upon binding double-stranded DNA. The housekeeping gene $\beta$-actin was used as the internal control. All primers were purchased from Takara Bio, Inc. cDNA was subjected to denaturing $\left(95^{\circ} \mathrm{C}, 30 \mathrm{sec}\right)$, annealing $\left(55^{\circ} \mathrm{C}, 15 \mathrm{sec}\right)$, and extension $\left(95^{\circ} \mathrm{C} 5 \mathrm{sec}\right.$ and $60^{\circ} \mathrm{C} 30 \mathrm{sec}$ ) for 40 cycles. The relative expression of CHFR mRNA was quantified using the $2^{-\Delta \Delta C q}$ method (16). PCR amplification was performed using the following primers: CHFR forward, 5'-CAGCTTCCGTGAGCTGACCTATC-3' and reverse, 5'-GCGTGGTGAGCTTTCACCTG-3'; and $\beta$-actin forward, 5'-TGGCACCCAGCACAATGAA-3' and reverse, 5'-CTAAGTCATAGTCCGCCTAGAAGCA-3'.
Western blot analysis. Cells were lysed on ice using radioimmunoprecipitation assay lysis buffer (Beyotime Institute of Biotechnology, Shanghai, China). Cell lysates were quantified by a BCA Protein Assay kit (\#P0010S; Beyotime Institute of Biotechnology) and equal amounts of cell protein were loaded into each well of $10 \%$ SDS-PAGE gels. After electrophoresis, proteins on gels were transferred to polyvinylidene difluoride membranes (Merck Millipore, Darmstadt, Germany). Membranes were blocked with 5\% (w/v) non-fat milk and then incubated with primary antibodies (rabbit anti-human CHFR polyclonal antibody, \#4297S; dilution, 1:1,000; Cell Signaling Technology, Inc., Boston, MA, USA; and mouse anti-human FLAG monoclonal antibody, \#F1804; dilution, 1:3,000; Sigma-Aldrich Co. LLC., Santa Clara, CA, USA) overnight at $4^{\circ} \mathrm{C}$. Membranes were washed four times, and then incubated with goat anti-rabbit or anti-mouse secondary antibodies (goat anti-rabbit, \#sc-2004; and goat anti-mouse, \#sc-2005; dilution, 1:5,000; both from Santa Cruz Biotechnology, Inc., Dallas, TX, USA). The membranes were the incubated in ECL western blot substrates (\#M3121/1859022; Thermo Fisher Scientific, Inc.) for $1 \mathrm{~min}$, and exposed to X-ray film. $\beta$-actin was used as a control.

Cell proliferation, migration and invasion assays. For cell proliferation assay, Capan-1_CHFR or Capan-1_Ctrl cells $\left(6 \times 10^{3} / 100 \mu \mathrm{l}\right)$ were seeded in 96 -well plates and cultured for $0,24,48,72$ and $96 \mathrm{~h}$ at $37^{\circ} \mathrm{C}$. Next, $100 \mu \mathrm{l}$ serum-free RPMI-1640 containing 10\% Cell Counting Kit-8 (CCK-8) reagent (Dojindo, Tokyo, Japan) was added in each well, and cells were incubated for $4 \mathrm{~h}$ at $37^{\circ} \mathrm{C}$. Optical density values (OD) were read at $450 \mathrm{~nm}$ using a 96 -well plate reader (Thermo Fisher Scientific, Inc.). Transwell assays were performed to assess cell migration and invasion. For migration assays, Capan-1_CHFR or Capan-1_Ctrl cells (4x10 4 cells) were seeded into the upper chambers of the inserts with non-coated membrane (24-well insert; $8-\mu \mathrm{m}$ pore size; Corning Inc., Corning, NY, USA). For invasion assays, $4 \times 10^{4}$ cells were plated in the upper chambers with Matrigel-coated membrane (Corning Inc.). $100 \mu \mathrm{l}$ serum-free RPMI-1640 was added to the upper chamber and $600 \mu 1$ RPMI-1640 medium containing $10 \%$ FBS was added to the lower chamber. Cells were incubated for $48 \mathrm{~h}$ at $37^{\circ} \mathrm{C}$ in a $5 \% \mathrm{CO}_{2}$ atmosphere. The cells were then fixed in $4 \%$ paraformaldehyde solution for $30 \mathrm{~min}$ and stained with $5 \%$ crystal violet solution for $5 \mathrm{~min}$. Cells that invaded through the pores to the lower surface of the inserts were photographed and counted under an inverted microscope (Olympus Corporation, Tokyo, Japan).

Flow cytometric analysis. For cell cycle analysis, cells were cultured to logarithmic phase, harvested and fixed in ice-cold $70 \%$ ethanol overnight at $4^{\circ} \mathrm{C}$. Cells were then incubated with RNase A at $37^{\circ} \mathrm{C}$ for $30 \mathrm{~min}$ and stained with propidium iodide (\#KGA512; Nanjing KeyGen Biotech., Co., Ltd., Nanjing, China) for $30 \mathrm{~min}$ in the dark. The cellular DNA content and cell cycle phase distribution were analyzed with a FACS Caliber instrument (BD Biosciences, Franklin Lakes, NJ, USA). Apoptotic cells were detected by flow cytometry using the Annexin V-APC/7-AAD Apoptosis Detection kit (\#KGA1026; Nanjing KeyGen Biotech., Co., Ltd.). Capan-1_CHFR or Capan-1_Ctrl cells were inoculated into $25 \mathrm{~cm}^{2}$ culture flasks 
$\left(8 \times 10^{5}\right.$ cells/flask). After cells adhered, they were treated with 100,200 , or $400 \mathrm{nM}$ gemcitabine or 2,4 , or $8 \mathrm{nM}$ docetaxel (Sigma-Aldrich Co. LLC.) for $24 \mathrm{~h}$ and then harvested. The staining was performed according to the manufacturer's instructions. Flow cytometry evaluation of the apoptotic rate was performed using a FACS Caliber instrument (BD Biosciences). The percentage of specific apoptosis was calculated using the following equation: $\%$ specific apoptosis $\%=\{1-[100 \%-\%$ $\left(\right.$ Annexin $\mathrm{V}^{+}+$Annexin $\mathrm{V}$ 7-AAD $\left.\left.{ }^{+}\right)\right]-[\%$ spontaneous $\left(\right.$ Annexin $\mathrm{V}^{+}+$Annexin $\mathrm{V}$ 7-AAD $\left.\left.{ }^{+}\right)\right]$. Three wells were assessed at each condition and the experiment was repeated three times.

Patients and tissue specimens. The study was approved by the Ethics Committee of Xuanwu Hospital, Capital Medical University (Beijing, China) in accordance with the guidelines for the protection of human subjects (17). Patients with PDAC who had undergone radical resection in General Surgery Department at Beijing Xuanwu Hospital between March 2008 and October 2015 were retrospectively identified via medical records and pathology reports. None of the patients received chemotherapy or radiation therapy prior to cancer resection. All tissue samples were obtained following informed consent. Selected hematoxylin and eosin-stained slides were reviewed by an experienced pathologist at Xuanwu Hospital to confirm the original pathological diagnoses and to choose representative areas. The pathologic specimens should contain both tumor tissues and adjacent non-tumor tissues. All tumors were staged according to the pathological tumor-node-metastasis (TNM) staging system of the American Joint Committee on Cancer.

Immunohistochemistry. Twenty-seven PDAC samples and paired non-tumor tissue samples were immunohistochemically stained by the Envision method. The sections $(5 \mu \mathrm{m}$ thick) were deparaffinized, rehydrated with graded concentrations of ethanol, and incubated with $3 \% \mathrm{H}_{2} \mathrm{O}_{2}$ for $15 \mathrm{~min}$. The sections were then incubated with the primary antibody (mouse anti-human CHFR monoclonal antibodies, \#H00055743-M01; dilution, 1:100; Abnova, Taibei, Taiwan) in a moist chamber (dark, $4^{\circ} \mathrm{C}$, overnight), followed by incubation with secondary antibodies (anti-mouse/rabbit, \#KIT-9922; Fuzhou Maixin Biotech Co., Ltd., Fuzhou, China) for $15 \mathrm{~min}$ at room temperature and DAB reagent (Fuzhou Maixin Biotech Co., Ltd.). The sections were then counterstained with hematoxylin and dehydrated. CHFR staining was scored both for nuclear and cytoplasmic staining based on intensity ( 0 , no staining; 1 , weak staining; 2 , strong staining) and percentage of cells stained $(0$, $<10 \% ; 1,10-50 \% ; 2,>50 \%)(15,18)$. Scores for intensity and percentage of stained cells were added for a maximum score of 4 . Scores of 4 were considered 'high' expression, while all others were 'low' expression. Immunohistochemistry was reviewed for accuracy of diagnosis and for scoring by an experienced pathologist who was blinded to the clinical outcomes of the patients.

Statistical analysis. Statistical analysis was performed using SPSS version 20.0 (IBM Corp., Armonk, NY, USA). A Mann-Whitney U test (comparisons between two groups) was used for analysis and $\mathrm{P} \leq 0.05$ was considered to indicate a statistically significant difference. The association of CHFR expression with histological or clinical factors was analyzed using Chi-square test or Fisher's exact tests. Kaplan-Meier and time series tests (log-rank test) were used for univariate survival analysis.

\section{Results}

CHFR expression in pancreatic cancer cells. We first examined endogenous CHFR mRNA and protein levels in five human pancreatic cancer cell lines, CFPAC-1, BxPC-3, Capan-1, PANC-1, and SW1990, using RT-qPCR and western blot analysis. The results showed that CHFR levels varied across all cell lines (Fig. 1A and B). Capan-1 cells expressed the lowest level of CHFR, and thus we selected these cells for further analyses. We next established Capan-1 cells with stable overexpression of CHFR by lentiviral infection; Capan-1_Ctrl cells were established as controls (Fig. 1E). We confirmed that CHFR mRNA levels were significantly upregulated in the Capan-1_CHFR cells compared to the Capan-1_Ctrl cells $(\mathrm{P}<0.01$; Fig. 1D). Exogenous CHFR protein expression was also confirmed in Capan-1_CHFR cells, but not in control cells (Fig. 1C).

Effects of CHFR overexpression on cell proliferation and cell cycle. As shown in Fig. 2A, the proliferation of Capan-1_CHFR cells was significantly inhibited at 48,72 and $96 \mathrm{~h}$ compared to Capan-1_Ctrl cells $(\mathrm{P}<0.01)$. To evaluate the mechanism underlying the inhibitory effect of CHFR in more detail, we examined the cell cycle phase distribution of Capan-1 stable cell lines using flow cytometry after staining with PI. Interestingly, the results showed that the percentage of cells in the G1 phase was significantly increased in Capan-1_CHFR cells compared to Capan-1_Ctrl cells ( $\mathrm{P}<0.01$; Fig. 2B and C). These results indicated that CHFR inhibited the proliferation of Capan-1 cells by arresting cells at G1 phase.

Effects of CHFR overexpression on cell migration and invasion. Cell migration and invasion abilities were next determined by Transwell assays. We found that, compared with Capan-1_Ctrl cells, Capan-1_CHFR cell migration was significantly reduced $(\mathrm{P}<0.01)(\mathrm{Fig} .2 \mathrm{D}$ and $\mathrm{F})$. The Transwell invasion assays also showed that cell invasion was inhibited in Capan-1_CHFR cells compared to control cells $(\mathrm{P}<0.05)$ (Fig. 2D and G).

Effect of CHFR overexpression on apoptosis of Capan-1 cells. We next evaluated the apoptotic response of Capan-1_CHFR and Capan-1_Ctrl cells by treating cells with different concentrations of gemcitabine or docetaxel for $24 \mathrm{~h}$ (Fig. 2E and H). Before formal experiments, we have tried different action times of the drugs. When the action time was prolonged to $48 \mathrm{~h}$, in the both groups treated with gemcitabine at the concentrations of $400 \mathrm{nM}$, there were more than $70 \%$ cells in the right upper quadrant (Annexin V 7-AAD ${ }^{+}$, which contained both necrosis and the late stage apoptotic cells (data not shown). When the action time was prolonged to $72 \mathrm{~h}$, after two times of washing with PBS, the rest cells in the tubes were even not enough to be analyzed with the instrument. Besides, we also tried the time point shorter than 
A

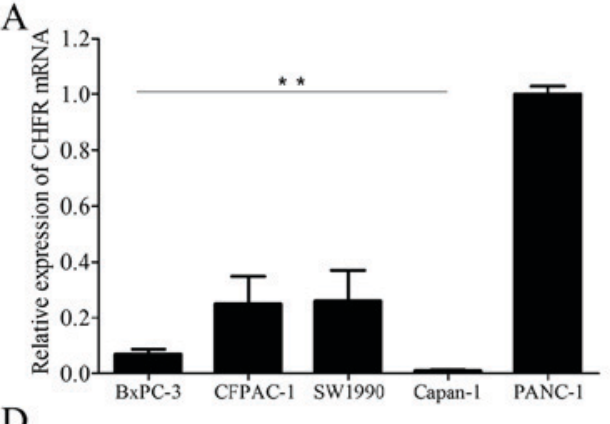

$\mathrm{D}$

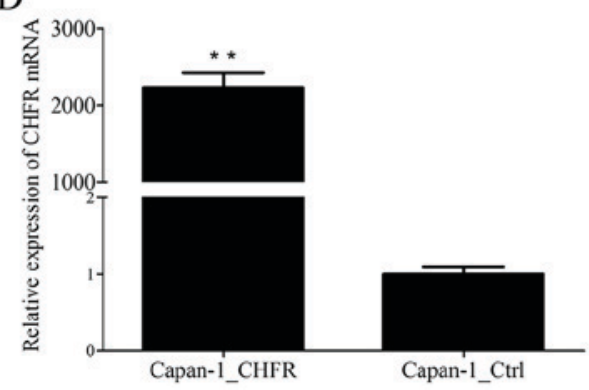

B

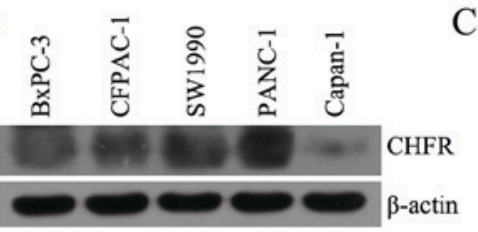

$\mathrm{C}$

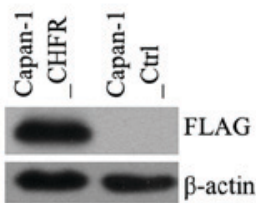

E
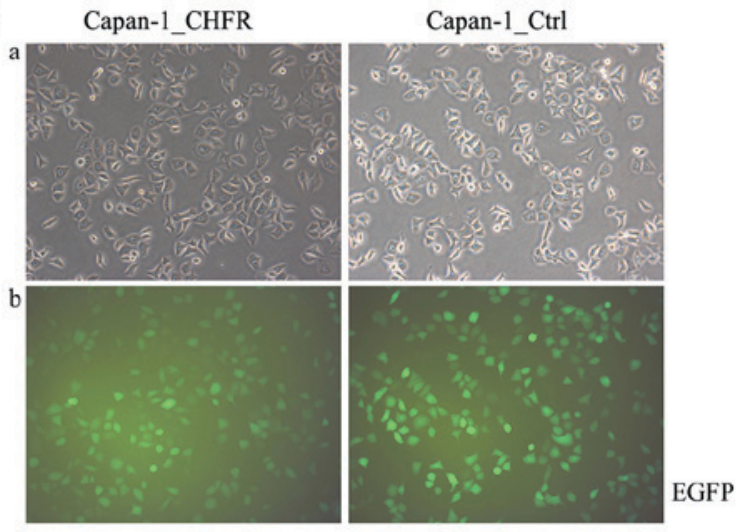

Figure 1. Expression of CHFR in pancreatic cancer cell lines. (A) Endogenous CHFR mRNA expressions in five human pancreatic cancer cell lines, BxPC-3, CFPAC-1, SW1990, Capan-1, and PANC-1 were determined by RT-qPCR. ${ }^{* *} \mathrm{P}<0.01$. (B) CHFR protein expression in five human pancreatic cancer cell lines, BxPC-3, CFPAC-1, SW1990, PANC-1 and Capan-1 were determined by western blotting. (C) Exogenous CHFR protein expression in Capan-1_CHFR and Capan-1_Ctrl cells were determined by western blotting. (D) CHFR mRNA expressions in Capan-1_CHFR and Capan-1_Ctrl cells were determined by RT-qPCR. ${ }^{* *} \mathrm{P}<0.01$. (E) After transfection, Capan-1_CHFR and Capan-1_Ctrl cells were observed by (a) inverted optical microscopy and (b) inverted fluorescence microscopy. Note that EGFP fluorescence could be observed in most of the cells, which is indicative of successful transfection. Original magnification, x200. CHFR, checkpoint with forkhead and ring-finger; RT-qPCR, reverse transcription-quantitative polymerase chain reaction; EGFP, enhanced green fluorescent protein.

$24 \mathrm{~h}$. When the action time was $12 \mathrm{~h}$, the apoptosis rates of the both groups were too close to the basal apoptotic level at the concentration of docetaxel $4 \mathrm{nM}$ (data not shown). Therefore, we chose the time point of $24 \mathrm{~h}$ for our formal experiments. The results showed that calculated with the equation mentioned in Materials and methods, a decreased apoptotic rate was detected in Capan-1_CHFR cells treated with docetaxel at the concentrations of 4 and $8 \mathrm{nM}$ compared with Capan-1_Ctrl cells. However, no statistically significant differences in apoptosis were observed in response to gemcitabine treatment $(\mathrm{P}<0.05 ; \mathrm{P}<0.01)($ Fig. $2 \mathrm{H})$.

Correlation between CHFR immunohistochemical expression and clinicopathological parameters. Considering the lasting time span of the PDAC cases, some of the pathological data were not intact, while some patients lost to follow-up. Only 27 patients were finally included in our research. The patients' ages ranged from 48 to 79 years (median, 64 years). The median follow-up period was 16.9 months (range, 0-59 months). The patients' clinicopathological data are summarized in Table I. Fig. 3A shows the representative samples of (panels a and b) CHFR 'low' and (panel c) 'high' expression in tumor and adjacent non-tumor tissues. Among the total 27 PDAC tumor samples, CHFR low expression was observed in 14 samples (51.9\%) and CHFR high expression was observed in 13 samples (48.1\%). Compared with adjacent non-tumor tissues, CHFR expression level was significantly increased in tumor tissue samples $(\mathrm{P}<0.05)$. We next analyzed the relationship between CHFR expression level and clinicopathologic factors. Interestingly, there was a striking significant correlation between high CHFR expression and earlier T-stage
$(\mathrm{P}=0.016)$ (Table I). Then we performed Kaplan-Meier survival curve analysis for the patients according to CHFR expression level. However, the log-rank test demonstrated no statistically significant differences between overall survival and CHFR expression levels ( $\mathrm{P}=0.102$; Fig. 3B). The 5-year survival rate was $0 \%$.

\section{Discussion}

CHFR was initially identified as a member of a small family of proteins that contain the FHA and RING-finger domains. This protein functions as a cell cycle checkpoint regulator, and thus we named it CHFR. The FHA domain of CHFR was confirmed to be responsible for its anti-proliferative effects (5), while the RING-finger domain has proven to be related to the mitotic checkpoint function (19). E3 ubiquitin ligase activity of CHFR and its autoubiquitination process also requires the RING-finger domain $(20,21)$. In addition, CHFR contains a unique PBZ motif in the C-terminal cysteine-rich region, which can not be found among other members of the FHA-RING protein family (22). However, the function of this motif remains largely unknown.

It was the first time to discuss the relationship between CHFR and pancreatic cancers. Our CCK- 8 proliferation assays showed that the proliferation of Capan-1_CHFR cells was significantly inhibited compared to control cells. Flow cytometric analysis revealed Capan-1 cells stably overexpressing CHFR showed a significant increase in the G1 cell population, which may indicate that CHFR inhibited cell proliferation by arresting cell cycle at G1 phase. This is quite different from several studies that reported the mitotic checkpoint 
A

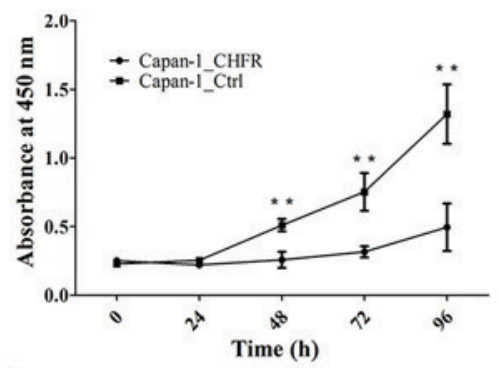

D
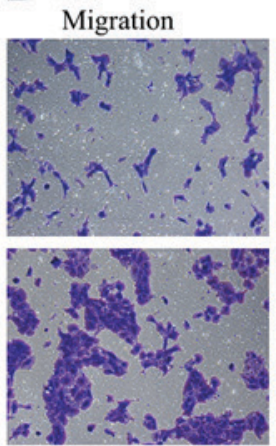

$\mathrm{F}$

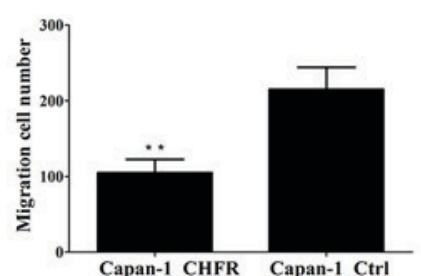

B

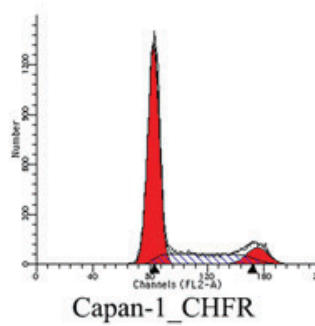

E
C

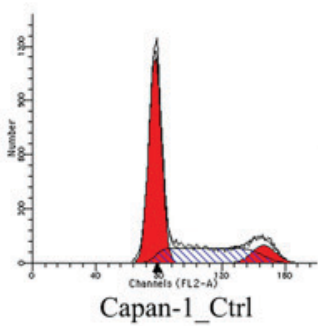

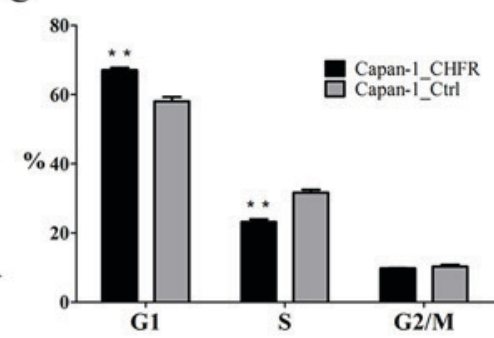
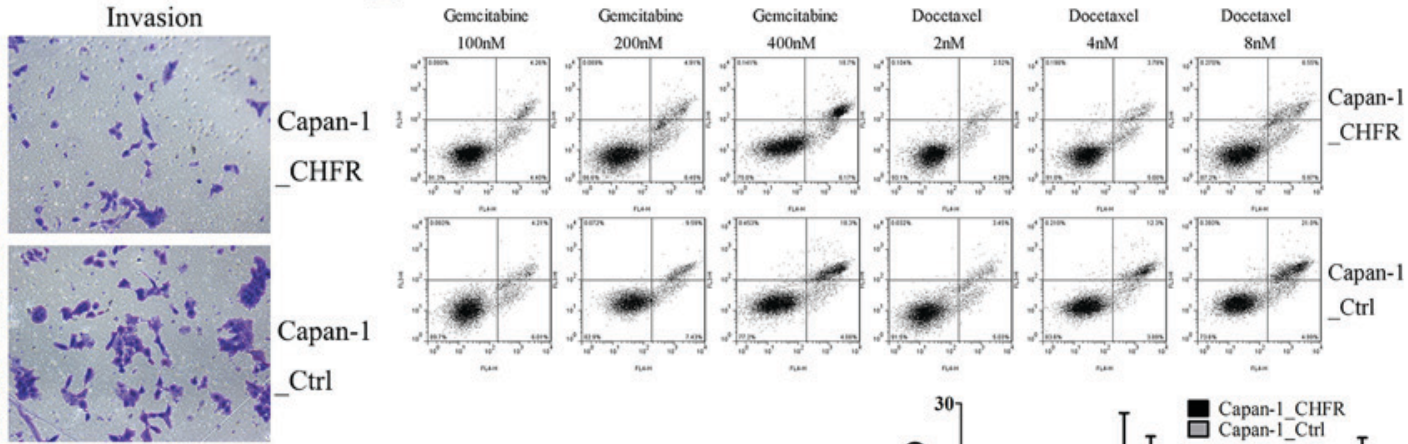

G

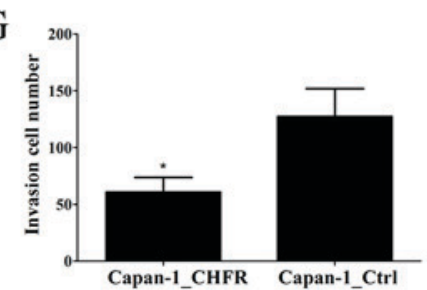

$\mathrm{H}$

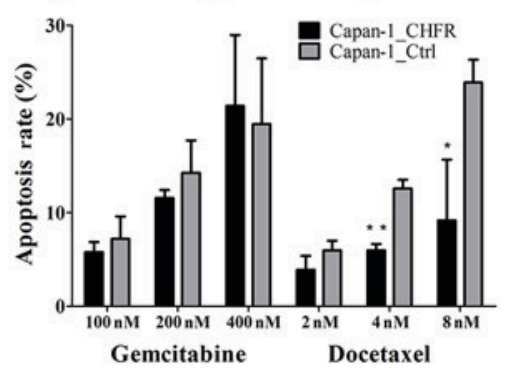

Figure 2. Effect of CHFR-overexpression in Capan-1 cells. (A) CCK-8 assays of Capan-1_CHFR and Capan-1_Ctrl cells at 0, 24, 48, 72 and 96 h. ${ }^{* *} \mathrm{P}<0.01$. (B) Flow cytometry analysis of cell cycle in Capan-1 cells after transfection. (C) Quantification of cell cycle phase distribution from flow cytometry analysis. ${ }^{* *} \mathrm{P}<0.01$. (D) Migration and invasion assays of Capan-1_CHFR and Capan-1_Ctrl cells in Transwell assays. Original magnification, $\mathrm{x} 200$. (E) Representative dot plots from flow cytometry illustrated apoptotic status of cells treated with different concentrations of gemcitabine or docetaxel. (F) Quantification of migration cell number from Transwell migration assays. ${ }^{* *} \mathrm{P}<0.01$. (G) Quantification of invasion cell number from Transwell invasion assays. ${ }^{*} \mathrm{P}<0.05$. (H) Quantification of total apoptotic rate from flow cytometry analysis of Capan-1_CHFR and Capan-1_Ctrl cells treated with different concentrations of gemcitabine or docetaxel. ${ }^{*} \mathrm{P}<0.05 ;{ }^{* *} \mathrm{P}<0.01$. CHFR, checkpoint with forkhead and ring-finger; CCK-8, Cell Counting Kit-8.

function of CHFR $(5,23,24)$. In these studies, in the presence of mitotic stress, which was induced by adding microtubule poisons such as nocodazole, colcemid, and taxanes, CHFR played a role as an early mitotic checkpoint in antephase and in the arrest of cells at the G2-to-M transition (22). However, some studies presented other data. Oh YM (11) used Hela CHFR-overexpression cells to show that CHFR may play a role in checkpoint function by cell cycle arrest in G1 phase. In the study of Chf1 and Chf2 in S. cerevisiae (25), the members of FHA-RING protein family, which were considered to be orthologous to CHFR protein, overexpression of either Chf protein led to growth retardation and a large increase in G1 cells. Our results are in agreement with the findings. This observation may mean that in the absence of mitotic stress, in pancreatic cancer cells, CHFR contributes to checkpoint function during interphase of the cell cycle rather than mitosis phase. A few reports have also indicated that CHFR may also control the cell cycle at anaphase of mitosis (10). In our Transwell assays, Capan-1_CHFR cells showed reduced migration and invasion abilities compared to Capan-1_Ctrl cells. Although the cell numbers had certain growth after cultured for $48 \mathrm{~h}$, proliferation of the cells was restricted by the limited bottom areas of the inserts. Therefore, we believed that the exogenous CHFR should be the major factor to reduce the migration and invasion abilities of Capan-1_CHFR cells compared to control cells. Researches on the mechanism of this field indicated that, CHFR is able to inhibit the NF- $\kappa \mathrm{B}$ signaling pathway and IL-8, which subsequently resulted in decreased angiogenesis and cell migration $(26,27)$. Thus, we suggested that the inhibitory effects of CHFR on proliferative and metastatic activities in pancreatic cancer cells were consistent with previous findings in other kinds of cancers. However, as the specific checkpoint function of CHFR has not been clearly illustrated, it is possible it depends on cell specificity, and/or the expression level of relevant factors, such as Plk1 (28), Cyclin B1 (29), and Aurora A (30). The purpose of our study is to reveal the function of CHFR in pancreatic cancers. Our CCK-8 assay and flow cytometric analysis indicated that CHFR inhibited cell proliferation by arresting cell cycle at G1 phase. But the mechanism of this phenomenon has not been clearly illustrated. Inactive $\mathrm{Cdk} 1 / \mathrm{Cyclin} \mathrm{B} 1$ is a feature of the CHFR-dependent arrest state $(28,31,32)$. The major problem is identifying the direct protein targets of CHFR. Besides, a key protein that regulates the activity and translocation of cyclin B1 to the nucleus to initiate mitosis is Aurora A kinase (30). Therefore, Aurora A has also been 
Table I. Clinicopathological characteristics of pancreatic ductal adenocarcinoma cases by CHFR immunohistochemical expression.

\begin{tabular}{|c|c|c|c|c|}
\hline $\begin{array}{l}\text { Clinicopathological } \\
\text { characteristics }\end{array}$ & Total $\mathrm{N}=27(\%)$ & CHFR low $\mathrm{N}=14(\%)$ & CHFR high $\mathrm{N}=13(\%)$ & P-value \\
\hline Age (years) & & & & 0.706 \\
\hline$\geq 65$ & $13(48.1)$ & $6(42.9)$ & $7(53.8)$ & \\
\hline$<65$ & $14(51.9)$ & $8(57.1)$ & $6(46.2)$ & \\
\hline Sex & & & & 1.00 \\
\hline Male & $13(48.1)$ & $7(50)$ & $6(46.2)$ & \\
\hline Female & $14(51.9)$ & $7(50)$ & $7(53.8)$ & \\
\hline Site & & & & 0.440 \\
\hline Head & $17(63)$ & $10(71.4)$ & $7(53.8)$ & \\
\hline Body and tail & $10(37)$ & $4(28.6)$ & $6(46.2)$ & \\
\hline Maximum diameter of tumor, $\mathrm{cm}$ & & & & 1.00 \\
\hline$>4$ & $6(22.2)$ & $3(21.4)$ & $3(23.1)$ & \\
\hline$\leq 4$ & $21(77.8)$ & $11(78.6)$ & $10(76.9)$ & \\
\hline Differentiation & & & & 0.098 \\
\hline Well & $2(7.4)$ & $2(14.3)$ & $0(0)$ & \\
\hline Moderately & $23(85.2)$ & $10(71.4)$ & $13(100)$ & \\
\hline Poorly & $2(7.4)$ & $2(14.3)$ & $0(0)$ & \\
\hline T-stage & & & & 0.016 \\
\hline $\mathrm{T} 1-2$ & $5(18.5)$ & $0(0)$ & $5(38.5)$ & \\
\hline $\mathrm{T} 3-4$ & $22(81.5)$ & $14(100)$ & $8(61.5)$ & \\
\hline N-stage & & & & 0.449 \\
\hline NO & $14(51.9)$ & $6(42.9)$ & $8(61.5)$ & \\
\hline N1 & $13(48.1)$ & $8(57.1)$ & $5(38.5)$ & \\
\hline
\end{tabular}

CHFR, checkpoint with forkhead and ring-finger.

speculated to be a target for ubiquitination by CHFR. The interaction of these factors and CHFR will be pursued in our further studies.

Some tumors with CHFR deficiency show a better chemotherapeutic response to taxanes, such as paclitaxel and docetaxel $(14,15)$, which may indicate an aberrant mitosis checkpoint among these cancers. One probable cause of the phenomenon might be that cancer cells with intact CHFR expression possess an early mitotic checkpoint with normal function that delays their entry into mitosis, and microtubular damage repair was performed. In contrast, CHFR downregulation or deficient cells would enter mitosis with a non-functional mitotic checkpoint and thus undergo mitotic catastrophy, eventually resulting in apoptosis.

In clinical practice, gemcitabine is currently the first line chemotherapy for pancreatic adenocarcinoma, and taxanes, such as docetaxel and paclitaxel, have not shown superior clinical benefits compared with gemcitabine (33). For this reason, in addition to docetaxel, we also included gemcitabine in our study. We did not detect any difference in the basal apoptotic level of Capan-1_CHFR and Capan-1_Ctrl cells. However, Capan-1_CHFR cells showed a lower apoptosis rate upon treatment with the anti-microtubule agent docetaxel compared with the control cells, which was not observed with gemcitabine treatment. Therefore, we speculate that if the patients of pancreatic cancer were divided into different groups based on CHFR expression level, the CHFR low group might show a higher sensitivity to taxanes, and thus benefit more. Combined with the experimental results of proliferation, migration and invasion assays, we find an interesting dual character of CHFR. On one hand, overexpression of CHFR results in an inhibitory effect on multiple tumor malignant biological behaviors; on the other hand, CHFR downregulation might indicate a higher effective rate in response to anti-microtubule agents. This phenomenon fundamentally related to the cell cycle checkpoint function of CHFR. After radical resections, some of the patients may receive chemotherapy. When the formation of normal mitotic spindles was inhibited by docetaxel, CHFR downregulation cells would enter mitosis with a non-functional checkpoint, undergo mitotic catastrophy, and eventually resulting in apoptosis. Thus, treatment with docetaxel may benefit the patients with CHFR low expression. In vitro experiments, the use of only one cell line was the limitation of our present study. However, according to the finding of our research, as well as other studies reported previously $(11,25-27)$, we firmly believed that our conclusion was reliable. In our further study, we will try our best to improve the methods and break this limitation.

On the basis of published investigations, promoter $\mathrm{CpG}$ island methylation is the most common reason leading to CHFR inactivation (34). Besides, diminished CHFR expression also needed to be drew attention (15). So far, earlier diagnosis and treatment of PDAC is now still a grate problem for clinical 
A

b

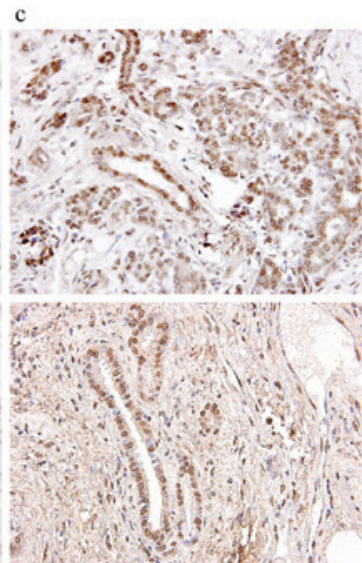

B

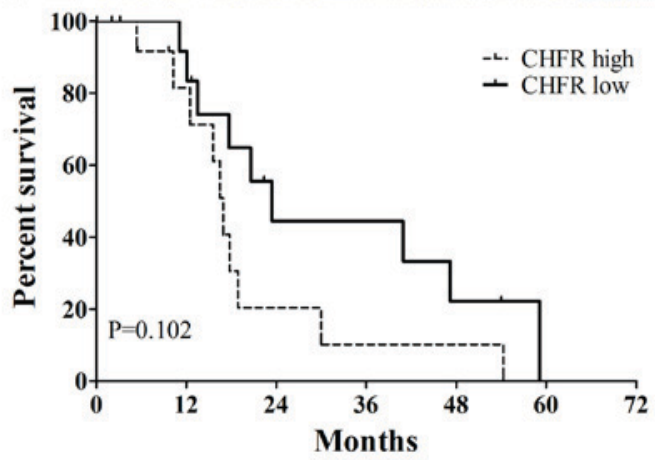

Figure 3. The functions of CHFR in PDAC patients. (A) Immunohistochemical analysis of CHFR expression in PDAC cases. Images show representative samples with scores of (a) ' 0 ', (b) ' 2 ' and (c) ' 4 ', in tumor tissues and adjacent non-tumor tissues. Tumors with scores of less than '4' are classified as samples with CHFR low expression. Original magnification, x400. (B) Kaplan-Meier plot of overall survival in patients with PDAC according to CHFR expression. $\mathrm{P}=0.102$. CHFR, checkpoint with forkhead and ring-finger; PDAC, pancreatic ductal adenocarcinoma.

practice. In the fact, most of the patients who came to see a doctor have already developed symptoms, which means the TNM stage was often not too early. Since all the cases involved in our study had undergone radical resection, most of them were at II or III stage when received operations. Patients at stage IV have lost the opportunities of radical surgery, while the detection of patients at I stage is really difficult. For these reasons, T1-2 case number was much less than T3-4. And it is the same case with the tumor size and differentiation. Smaller tumor size often means higher resection rate. Tumors with well differentiation and slow progress usually difficult to be discovered at an early stage. Patients with poorly differentiation tumor mostly lost the opportunity of surgical therapy because of early metastasis and rapid progress. Therefore, in our study, uneven distribution of the clinicopathological data might be inevitable. In the report of CHFR expression in malignant peripheral nerve sheath tumors in 2006 (18), the correlation between the immunohistochemical expression of CHFR and the clinicopathologic parameters was assessed by t-test, Chi-square test, and Fisher's exact test. In the data of tumor depth, tumor size and AJCC stage, there were big differences of case numbers in groups. In 2013, Pillai et al (15) reported CHFR protein expression in metastatic NSCLC. In the text, differences between CHFR high vs. low expression were assessed using ANOVA for numerical covariates and Chi-square test or Fisher's exact test for categorical variables, where appropriate. In the text, the sex distribution of cases was also very uneven (40 vs. 1). And it is the same case with the data of response. In 2014, Gebauer et al (35) studied the relationship between carcinoembryonic antigen-related cell adhesion molecules (CEACAM) and pancreatic cancer. For explorative statistical analysis of the individual patient groups, either a two-sided Chi-square test or a Fisher's exact test was used. There were also too much differences of case numbers between the groups of N0 and N1 (119 vs. 18). All the researches above used Chi-square test or Fisher's exact test for categorical variables. However, in the parts of discussion of these texts, we have not found any comment on the impact of uneven distribution of the data. In addition, we thought when we analyzed the correlation between CHFR expression and clinicopathologic features, what we really comparing was the groups of CHFR high and low expression, but not the groups of T1-2 and T3-4, or the tumor sizes. For these reasons, we believed that uneven distribution of clinicopathological data was ubiquitous in clinical researches, especially for malignant tumor, and the methods of statistical analysis we chose was appropriate. In PDAC patients, approximately half $(51.9 \%)$ of PDAC samples showed low expression level of CHFR in our study. Furthermore, we found a correlation between CHFR expression and T-stage, suggesting a certain connection between CHFR gene expression level and invasion ability of the tumors. This is consistent with our in vitro observations in invasion assays. Nevertheless, when compared with adjacent non-tumor tissues, we were surprised to find CHFR expression level dramatically increased. This result is very different from some previous reports in other tumors $(18,36,37)$. In considering possible reasons, we speculate that single-nucleotide polymorphisms (SNPs) of CHFR may play a role. In the initial report of CHFR in 2000 (5), the authors examined five cancer cell lines and found that the U2OS cell line contains 
a variation in the CHFR gene in the non-coding strand. This variation could be a mutation, because it occurred within a CG dinucleotide, which is a mutagenesis hot-spot. Further experiments confirmed that this variation led to the loss of partial functions of CHFR, but had no effect on its protein expression. Although few mutations of the CHFR gene have been detected in human tumor cells, several SNPs have been reported $(12,31,38)$. Therefore, we speculate in pancreatic cancer tissues, there might exist some SNPs, which affect partial function of CHFR, but do not affect the expression level of CHFR protein. Finally, although our survival analysis demonstrated no relationship between CHFR expression level and overall survival, as shown in Fig. 3B, patients with CHFR high expression tended to show a poorer survival rate compared to CHFR-low expression patients (although $\mathrm{P}=0.102$ ). We think there may be two possibilities for this phenomenon. Firstly, after radical resections, some of the patients might receive chemotherapy. In the chemotherapy regimens which included docetaxel, CHFR expression level might be able to influence the treatment effects, and affect the patients' prognosis. Secondly, as mentioned previously, SNPs or mutations of CHFR may exist in pancreatic cancers, which caused the loss of partial functions of the gene, and even became an unbeneficial factor of the patients' outcome. However, there has not been a consistent conclusion in this field. In metastatic NSCLC (16) diminished CHFR expression predicted a better prognosis, but CHFR inactivation in stage II colorectal cancer (39) and acute myeloid leukemia (40) were associated with an adverse outcome. In our study, although a significant association between CHFR expression and prognosis of PDAC patients was not observed, further studies with a larger sample size and more clinicopathological data are required to reveal deeper relationship between CHFR and PDAC.

In conclusion, our data show that $\mathrm{CHFR}$ regulates the cell cycle of pancreatic cancer cells by G1 phase arrest and it also functions as an important tumor suppressor in vitro. Nevertheless, CHFR overexpression is not a favorable factor in apoptosis induced by microtubule inhibitors. In PDAC patients, aberrant high expression of CHFR in tumor tissues compared with adjacent non-tumor tissues may not play its role effectively. CHFR expression level was significantly associated with the pathological stage; however, it is insufficient to function as a biomarker in prognosis. In our follow-up study, we will do more research on the mechanisms of CHFR in pancreatic cancer and the interaction of relevant factors. In addition, in vivo experiments are necessary to expand our current findings.

\section{Acknowledgements}

This study was supported by the National Natural Science Foundation of China (no. 81272756) and Beijing Natural Science Foundation (no. 7162076). The authors would like to acknowledge L.H. Teng for writing assistance, and Y.P. Zhang for proof reading the article.

\section{References}

1. Ilic M and Ilic I: Epidemiology of pancreatic cancer: World J Gastroenterol 22: 9694-9705, 2016.

2. Pannala R, Leirness JB, Bamlet WR, Basu A, Petersen GM and Chari ST: Prevalence and clinical profile of pancreatic cancer-associated diabetes mellitus. Gastroenterology 134: 981-987, 2008
3. Greenhalf W, Grocock C, Harcus M and Neoptolemos J: Screening of high-risk families for pancreatic cancer. Pancreatology 9: 215-222, 2009.

4. Shin EJ and Canto MI: Pancreatic cancer screening. Gastroenterol Clin North Am 41: 143-157, 2012.

5. Scolnick DM and Halazonetis TD: Chfr defines a mitotic stress checkpoint that delays entry into metaphase. Nature 406: 430-435, 2000.

6. Hofmann K and Bucher P: The FHA domain: A putative nuclear signalling domain found in protein kinases and transcription factors. Trends Biochem Sci 20: 347-349, 1995.

7. Lovering R, Hanson IM, Borden KL, Martin S, O'Reilly NJ, Evan GI, Rahman D, Pappin DJ, Trowsdale J and Freemont PS: Identification and preliminary characterization of a protein motif related to the zinc finger. Proc Natl Acad Sci USA 90: 2112-2116, 1993.

8. Borden KL, Boddy MN, Lally J, O'Reilly NJ, Martin S, Howe K, Solomon E and Freemont PS: The solution structure of the RING finger domain from the acute promyelocytic leukaemia proto-oncoprotein PML. EMBO J 14: 1532-1541, 1995.

9. Durocher D and Jackson SP: The FHA domain. FEBS Lett 513: 58-66, 2002.

10. Yu X, Minter-Dykhouse K, Malureanu L, Zhao WM, Zhang D, Merkle CJ, Ward IM, Saya H, Fang G, van Deursen J and Chen J: Chfr is required for tumor suppression and aurora a regulation. Nat Genet 37: 401-406, 2005.

11. Oh YM, Kwon YE, Kim JM, Bae SJ, Lee BK, Yoo SJ, Chung CH, Deshaies RJ and Seol JH: Chfr is linked to tumour metastasis through the downregulation of HDAC1. Nat Cell Biol 11: 295-302, 2009.

12. Bertholon J, Wang Q, Falette N, Verny C, Auclair J, Chassot C, Navarro C, Saurin JC and Puisieux A: Chfr inactivation is not associated to chromosomal instability in colon cancers. Oncogene 22: 8956-8960, 2003.

13. Yoshida K, Hamai Y, Suzuki T, Sanada Y, Oue N and Yasui W: DNA methylation of CHFR is not a predictor of the response to docetaxel and paclitaxel in advanced and recurrent gastric cancer. Anticancer Res 26: 49-54, 2006.

14. Yanokura M, Banno K, Kawaguchi M, Hirao N, Hirasawa A, Susumu N, Tsukazaki K and Aoki D: Relationship of aberrant DNA hypermethylation of CHFR with sensitivity to taxanes in endometrial cancer. Oncol Rep 17: 41-48, 2007.

15. Pillai RN, Brodie SA, Sica GL, Shaojin Y, Li G, Nickleach DC, Yuan L, Varma VA, Bonta D, Herman JG, et al: CHFR protein expression predicts outcomes to taxane-based first line therapy in metastatic NSCLC. Clin Cancer Res 19: 1603-1611, 2013.

16. Livak KJ and Schmittgen TD: Analysis of relative gene expression data using real-time quantitative PCR and the 2(-Delta Delta C(T)) method. Methods 25: 402-408, 2001.

17. Ketefian S: Ethical considerations in research. Focus on vulnerable groups. Invest Educ Enferm 33: 164-172, 2015.

18. Kobayashi C, Oda Y, Takahira T, Izumi T, Kawaguchi K, Yamamoto H, Tamiya S, Yamada T, Iwamoto Y and Tsuneyoshi M: Aberrant expression of CHFR in malignant peripheral nerve sheath tumors. Mod Pathol 19: 524-532, 2006.

19. Chin CF and Yeong FM: Safeguarding entry into mitosis: The antephase checkpoint. Mol Cell Biol 30: 22-32, 2010.

20. Bothos J, Summers MK, Venere M, Scolnick DM and Halazonetis TD: The Chfr mitotic checkpoint protein functions with Ubc13-Mms2 to form Lys63-linked polyubiquitin chains. Oncogene 22: 7101-7107, 2003.

21. Chaturvedi P, Sudakin V, Bobiak ML, Fisher PW, Mattern MR, Jablonski SA, Hurle MR, Zhu Y, Yen TJ and Zhou BB: Chfr regulates a mitotic stress pathway through its RING-finger domain with ubiquitin ligase activity. Cancer Res 62: 1797-1801, 2002.

22. Brooks L III, Heimsath EG Jr, Loring GL and Brenner C: FHA-RING ubiquitin ligases in cell division cycle control. Cell Mol Life Sci 65: 3458-3466, 2008.

23. Summers MK, Bothos J and Halazonetis TD: The CHFR mitotic checkpoint protein delays cell cycle progression by excluding Cyclin B1 from the nucleus. Oncogene 24: 2589-2598, 2005.

24. Ogi K, Toyota M, Mita H, Satoh A, Kashima L, Sasaki Y, Suzuki H, Akino K, Nishikawa N, Noguchi M, et al: Small interfering RNA-induced CHFR silencing sensitizes oral squamous cell cancer cells to microtubule inhibitors. Cancer Biol Ther 4: 773-780, 2005.

25. Bieganowski P, Shilinski K, Tsichlis PN and Brenner C: Cdc123 and checkpoint forkhead associated with RING proteins control the cell cycle by controlling eIF2gamma abundance. J Biol Chem 279: 44656-44666, 2004. 
26. Kashima L, Toyota M, Mita H, Suzuki H, Idogawa M, Ogi K, Sasaki Y and Tokino T: CHFR, a potential tumor suppressor, downregulates interleukin-8 through the inhibition of NF-kappaB. Oncogene 28: 2643-2653, 2009.

27. Lee KH, Bae SH, Lee JL, Hyun MS, Kim SH, Song SK and Kim HS: Relationship between urokinase-type plasminogen receptor, interleukin-8 gene expression and clinicopathological features in gastric cancer. Oncology 66: 210-217, 2004.

28. Kang D, Chen J, Wong J and Fang G: The checkpoint protein Chfr is a ligase that ubiquitinates Plk1 and inhibits Cdc2 at the G2 to M transition. J Cell Biol 156: 249-259, 2002.

29. Hagting A, Jackman M, Simpson K and Pines J: Translocation of cyclin B1 to the nucleus at prophase requires a phosphorylation-dependent nuclear import signal. Curr Biol 9: 680-689, 1999.

30. Hirota T, Kunitoku N, Sasayama T, Marumoto T, Zhang D, Nitta M, Hatakeyama K and Saya H: Aurora A and an interacting activator, the LIM protein Ajuba, are required for mitotic commitment in human cells. Cell 114: 585-598, 2003.

31. Matsusaka T and Pines J: Chfr acts with the $\mathrm{p} 38$ stress kinases to block entry to mitosis in mammalian cells. J Cell Biol 166 507-516, 2004.

32. Erson AE and Petty EM: CHFR-associated early G2/M checkpoint defects in breast cancer cells. Mol Carcinog 39: 26-33, 2004

33. Awasthi N, Zhang C, Schwarz AM, Hinz S, Schwarz MA and Schwarz RE: Enhancement of nab-paclitaxel antitumor activity through addition of multitargeting antiangiogenic agents in experimental pancreatic cancer. Mol Cancer Ther 13: 1032-1043, 2014.

34. Toyota M, Sasaki Y, Satoh A, Ogi K, Kikuchi T, Suzuki H, Mita H, Tanaka N, Itoh F, Issa JP, et al: Epigenetic inactivation of CHFR in human tumors. Proc Natl Acad Sci USA 100 7818-7823, 2003.
35. GebauerF, Wicklein D, Horst J, Sundermann P, Maar H, Streichert T, Tachezy M, Izbicki JR, Bockhorn $M$ and Schumacher U: Carcinoembryonic antigen-related cell adhesion molecules (CEACAM) 1, 5 and 6 as biomarkers in pancreatic cancer. PLoS One 9: e113023, 2014.

36. Privette LM, González ME, Ding L, Kleer CG and Petty EM: Altered expression of the early mitotic checkpoint protein, CHFR, in breast cancers: Implications for tumor suppression. Cancer Res 67: 6064-6074, 2007.

37. Soutto M, Peng D, Razvi M, Ruemmele P, Hartmann A, Roessner A, Schneider-Stock R and El-Rifai W: Epigenetic and genetic silencing of CHFR in esophageal adenocarcinomas. Cancer 116: 4033-4042, 2010.

38. Mariatos G, Bothos J, Zacharatos P, Summers MK, Scolnick DM, Kittas C, Halazonetis TD and Gorgoulis VG: Inactivating mutations targeting the chfr mitotic checkpoint gene in human lung cancer. Cancer Res 63: 7185-7189, 2003.

39. Cleven AH,Derks S, Draht MX,Smits KM,Melotte V, VanNesteL, Tournier B, Jooste V, Chapusot C, Weijenberg MP, et al: CHFR promoter methylation indicates poor prognosis in stage II microsatellite stable colorectal cancer. Clin Cancer Res 20: 3261-3271, 2014.

40. Gao L, Liu F, Zhang H, Sun J and Ma Y: CHFR hypermethylation, a frequent event in acute myeloid leukemia, is independently associated with an adverse outcome. Genes Chromosomes Cancer 55: 158-168, 2016. 